\title{
Research Paper: The Impact of Type 2 Diabetes Mellitus on Respiratory System
}

\author{
Bahar Yousefinezhadi $^{1}$ (D, Majid Ravanbakhsh ${ }^{*}$ (D), Maryam Saadat ${ }^{1}$ Mehrnoosh Zakerkish $^{2}$ (D), Shahin Goharpey ${ }^{1}$ (D) \\ 1. Research Center for Musculoskeletal Rehabilitation, Ahvaz Jundishapur University of Medical Sciences, Ahvaz, Iran. \\ 2. Health Research Institute, Diabetic Research Center, Ahvaz Jundishapur University of Medical Sciences, Ahvaz, Iran.
}

\begin{tabular}{l|l}
$\begin{array}{c}\text { Use your devic to scan } \\
\text { and read the article online }\end{array}$ \\
oftation Yousefinezhadi B, Ravanbakhsh M, Saadat M, Zakerkish M, Goharpey Sh. The Impact of Type 2 Diabetes Mellitus \\
on Respiratory System. Journal of Modern Rehabilitation. 2018; 12(3):157-162. http://dx.doi.org/10.32598/JMR.V12.N3.157
\end{tabular}

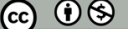

Article info:

Received: 13 Feb 2018

Accepted: 04 Jun 2018

Available Online: 01 Jul 2018

\section{Keywords:}

Diabetes Mellitus, Type 2, Capnography, Respiration, Lung

\begin{abstract}
A B S T RACT
Introduction: Type 2 diabetes is one of the major causes of progressive microvascular and macro vascular complications leading to retinopathy, nephropathy, and neuropathy as well pulmonary complications due to changes in collagen, elastin, and micro angiopathy. The current study aimed at assessing some respiratory indices in patients with type 2 diabetes and the possible relationships between these parameters and the level of blood glucose control and duration of diabetes.
\end{abstract}

Materials and Methods: In the current descriptive-analytical study, 20 patients with type 2 diabetes were compared with 20 matched healthy subjects. The information of the respiratory system was collected using a chronograph. Parameters for assessment were respiration rate, heartbeats, and oxygen saturation percentage.

Results: The results of the study showed a significant reduction in end-expiratory carbon dioxide pressure and increased respiratory rate in the patients compared to the control group. The results also showed a strong and inverse relationship between hemoglobin A1c (HbAlc) and respiratory indices. The duration of having diabetes had a negative significant relationship with respiratory indices.

Conclusion: In general, the results of the study indicated that lung function is impaired in people with type 2 diabetes. According to the results of the current study, a proposal for a program to facilitate or prevent lung function impairment for these patients is suggested.

\section{Introduction}

iabetes is one of the most common diseases of endocrine glands and the fourth cause of mortality in THE developed countries, with prevalence in the current century. It has increased by around $50 \%$ as compared to 10 previous years, and this number is projected to double by $2030[1,2]$. Diabetes is one of the diseases of metabolism, which develops due to different genetic or acquired causes [3]. In this disease, due to impaired secretion or activity of insulin, blood sugar rises, a condition known as hyperglycemia, causing impaired metabolism of carbohydrate, fat, and protein in these in-

\section{* Corresponding Author:}

Majid Ravanbakhsh, PhD.

Address: Research Center for Musculoskeletal Rehabilitation, Ahvaz Jundishapur University of Medical Sciences, Ahvaz, Iran.

Tel: +98 (61) 3367543

E-mail: majidphdpt@yahoo.com 
dividuals [4]. Type II diabetes (insulin resistant), which accounts for $90-95 \%$ of cases of diabetes is a result of progressive impairment in insulin secretion [5].

The quality of life of people with diabetes is often affected by the chronic complications of diabetes, which include micro- and macro angiography, retinopathy, neuropathy, nephropathy, phrenic nerve damage, increased probability of respiratory infections, diminished basic tone of bronchi, increased incidence of obstructive sleep apnea, and diminished cough reflex sensitivity [4]. These complications are contingent upon the duration of having diabetes.

Respiration plays a significant role in the body health and in maintaining the general homeostasis of the body through altering oxygen arterial pressure, carbon dioxide, blood PH, and Cerebrospinal Fluid (CSF). Carbon dioxide plays a vital role in keeping the basic acid balance of the body, and also affects different systems of the body considerably through fine and minor changes in the acid-base balance. In response to changes of $\mathrm{pH}$, the body is forced to alter the respiration rate and in turn the respiration depth and other respiratory parameters $[6$, 7]. Any disorder in the respiration can lead to altered respiratory pattern and incidence of impaired respiratory pattern [8].

A wide capillary network in the lungs might be affected by diabetes related to micro angiopathy and impaired pulmonary function, which is often neglected [9]. Some studies show diminished pulmonary function in adults with diabetes, as compared with non-diabetic individuals. Meanwhile, these studies assess volumetric parameters of lung such as Forced Vital Capacity (FVC) and Forced Expiratory Volume in one second (FEV1) using a spirometer $[10,11]$. Also, this diminished function in the lungs shows an inverse relationship with the blood sugar level and duration of having diabetes $[12,13]$.

Chronography is a non-invasive method that measures the pressure of the end tidal carbon dioxide by infrared light [14]. Although arterial blood gas test measures the amount of arterial carbon dioxide, it is painful and affected by momentary changes of respiratory parameters. Chronography is a good criterion to express the level of alveolar carbon dioxide under normal cardiac and pulmonary function [15]. In this regard, a good agreement is reported between chronography and Arterial Blood Gas (ABG) analysis [16].

Previous studies on respiratory assessment of patients with diabetes focused on volumetric findings of lungs using a spirometer. Nevertheless, to the best of authors knowledge, no investigations examined the pressure of the carbon dioxide in patients with type 2 diabetes. Therefore, the current study aimed at investigating the end tidal carbon dioxide pressure, blood oxygenation percentage, and respiratory rate in patients with type 2 diabetes.

\section{Materials and Methods}

The current case-control study was conducted on 20 patients with type 2 diabetes and 20 healthy individuals within the age range of 35-55 years matched in terms of factors such as age, gender, and body mass index with the patient group. Subjects with diabetes were selected based on diagnosis of a physician, and individuals with the results obtained from the HbAlc test over 7.5 performed by the diabetes research center of Jundishapour University of Medical Sciences, Ahvaz, Iran. They were included in the study as individuals with improper control of blood sugar regulation [17].

First, the subjects were familiarized with the study design. If the subjects consented, they signed the consent form. Then, demographic data including age, height and weight were recorded and the subjects were asked to complete the Nijmegen questionnaire [18]. The information of the respiratory system was collected using a chronograph (model 2500, Viamed Co. England), belonging to the laboratory of musculoskeletal research center of Faculty of Rehabilitation. Before initiating any tests, the system was calibrated and the subjects were asked to sit on comfortable chairs, with a nasal cannula connected to the subjects' noses.

The individuals were assured that this test had no risk or caused no complication for them. The subjects were then asked to breathe through nose and avoid speaking or moving during the test. Oximeter pulse placed on the finger of the subjects was used to measure the oxygen saturation percentage and heartbeats. Information recording was performed three times for each subject. Also, 10 minutes was given to the subjects between each time of the test to open the cannula, walk for some minutes to prevent fatigue or any other confounding factors. Furthermore, the temperature of the experimental setting was controlled as it could influence the respiration.

\section{Statistical analysis}

For data analysis, SPSS version 23.0 (SPSS Inc. Chicago, IL, USA) for windows was employed. The Kolmogorov-Smirnov test was applied to find normal 
distribution of data .Independent t-test and the MannWhitney test were employed to investigate the differences between the two groups. Significance level of less than $5 \%$ was accepted. Furthermore, Pearson correlation analysis was utilized to find the extent of relationship between the respiration variables including End Tidal Carbon Dioxide pressure $\left(\mathrm{ETCO}_{2}\right)$, the respiratory rate, and the blood oxygen saturation percentage with the past three-month blood sugar levels Hemoglobin A1C and duration of having diabetes.

\section{Results}

The patients with diabetes and healthy individuals had no significant difference in terms of age, height, and weight. The demographic characteristics of the two groups are presented in Table 1. The KolmogorovSmirnov test showed normal distribution of the end-tidal carbon dioxide pressure, respiration rate, and heartbeats both in the diabetic and control groups. However, the distribution of oxygen saturation percentage was not normal in the two groups. The results of independent t-test and the Mann-Whitney test revealed a significant difference in the indicators of end-tidal carbon dioxide pressure and the rate of respirations per minute as compared to those of the control group (Figure 1).

Nevertheless, no significant difference was observed in the variables of oxygen saturation percentage and heartbeats between the two groups. The mean end-tidal carbon dioxide pressure of the patient group was $4 \mathrm{mmHg}$ less than that of the control group. Also, the mean respiration rate in the patient group was 2.4 respirations per minute more than that of the control group (Table 2). Pearson correlation analysis showed a strong inverse and significant correlation between $\mathrm{HbAlc}$ and end-tidal carbon dioxide pressure indicator $(\mathrm{r}=-0.62, \mathrm{P}=0.003)$.
Also, a strong positive and significant correlation was observed between the respiration rate and $\mathrm{HbAlc}$ $(\mathrm{r}=0.97, \mathrm{P}=0.02)$. However, no significant relationship was observed between HbAlc and oxygen saturation percentage and heartbeats. There was a negative significant relationship between end tidal carbon dioxide pressure and the duration of having diabetes $(r=-0.46$, $\mathrm{P}=0.03$ ). Finally, no significant relationship was observed between the duration of having diabetes and the respiration rate as well as oxygen saturation percentage and heartbeats.

\section{Discussion}

The current study aimed at investigating the respiration pattern and indicators measuring vital blood gases as well as the relationship between these indicators and $\mathrm{HbA} 1 \mathrm{c}$ plus the duration of having diabetes among the patients with type 2 diabetes. In the current study, a significant difference was observed in the end-tidal carbon dioxide pressure and respiration rate between the diabetic and healthy groups. In patients with diabetes, the end-tidal carbon dioxide pressure showed a significant reduction compared to those of the healthy individuals, while the respiration rate indicated a significant growth.

The possible mechanisms resulting from these changes in the diabetic group can be attributed to the micro angiography of alveolar tissue and capillaries leading to restriction of alveolar gas transport. Also, in diabetic patients, long-term contact of monosaccharides with collagen fibers causes formation of spontaneous bonds between sugar molecules and amino acids. These changes increase the thickness of collagen molecules as well as the pulmonary capillary network, thereby narrowing the alveolar- capillary membrane. These results were in line with the findings obtained by Zineldin et al., Fuso et al., and Singh et al. [13, 19, 20]. They reported dimin-

Table 1. Demographic characteristics of diabetic and control groups $(n=20)$

\begin{tabular}{|c|c|c|c|}
\hline \multirow{2}{*}{ Demographic Data } & \multicolumn{2}{|c|}{ Mean $\pm S D$} & \multirow{2}{*}{$\mathbf{P}$} \\
\hline & Diabetic & Control & \\
\hline Age (y) & $48.60 \pm 5.99$ & $48.55 \pm 4.66$ & 0.76 \\
\hline Height $(\mathrm{cm})$ & $164.40 \pm 8.56$ & $165.60 \pm 8.22$ & 0.91 \\
\hline Weight (kg) & $71.70 \pm 9.05$ & $72.20 \pm 8.78$ & 0.82 \\
\hline Time since disease $(y)$ & $10.20 \pm 4.90$ & N/A & N/A \\
\hline Body Mass Index $\left(\mathrm{kg} / \mathrm{m}^{2}\right)$ & $26.47 \pm 2.72$ & $26.36 \pm 3.09$ & 0.49 \\
\hline
\end{tabular}

N/A: Not applicable 
Table 2. Respiratory parameters of diabetic and control groups

\begin{tabular}{|c|c|c|c|}
\hline \multirow{2}{*}{ Variable } & \multicolumn{2}{|c|}{ Mean $\pm S D$} & \multirow{2}{*}{$\mathbf{P}$} \\
\hline & Diabetic & Control & \\
\hline $\mathrm{ETCO}_{2}(\mathrm{mmHg})$ & $30.70 \pm 0.47$ & $34.80 \pm 0.70$ & $<0.01$ \\
\hline RR (breath/min) & $17.77 \pm 2.04$ & $15.34 \pm 2.99$ & $<0.01$ \\
\hline $\mathrm{SPO}_{2}(\%)$ & $98.01 \pm 1.16$ & $97.83 \pm 1.11$ & 0.60 \\
\hline $\mathrm{HB}$ (beat/min) & $82.56 \pm 11.96$ & $75.74 \pm 8.83$ & 0.04 \\
\hline
\end{tabular}

$\mathrm{ETCO}_{2}$ : End-Tidal Carbon Dioxide pressure, RR: Respiratory Rate, $\mathrm{SPO}_{2}$ : Oxygen Saturation Percentage, HB: Heart Beats

$J \mathrm{MR}$

ished pulmonary volumes and end tidal carbon dioxide pressure in patients with type 2 diabetes compared with healthy individuals.

In the current study, no significant difference was observed between the healthy and diabetic groups in terms of blood oxygen saturation percentage. These results were incongruent with the findings obtained by Wheatley et al. [21]. They reported diminished oxygen saturation percentage among 12 patients with type 1 diabetes during vigorous exercise. The possible reason of this incongruence can be due to the position of individuals in the study. Specifically, it was performed in sitting position, while Wheatley et al. measured blood oxygenation percentage during exercise.

It seems that more vigorous situations such as performing an activity or doing exercise are more suitable to show the blood oxygen saturation percentage difference between diabetic and control groups. In this study diabetic patients had higher oxygen saturation percentage compared with control group. Elevated blood HbA1c levels lead to an overestimation of the oxygen saturation percentage. It is due to high blood sugar levels and increase the tendency of oxygen to bonding with hemoglobin chains. [22].

A

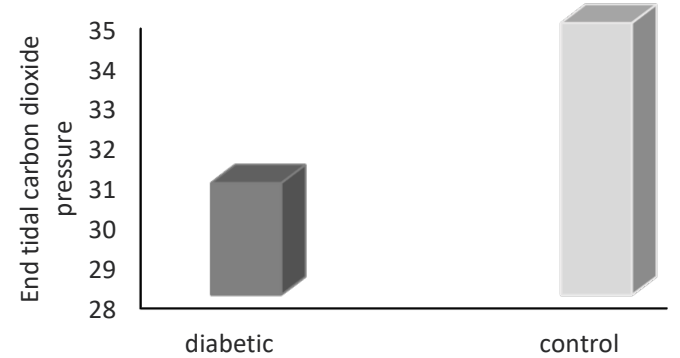

The study showed an inverse and strong significant relationship between $\mathrm{HbA} 1 \mathrm{c}$ and end tidal carbon dioxide pressure index $(\mathrm{r}=-0.62, \mathrm{P}=0.003)$. It also indicated an inverse and moderate correlation between carbon dioxide pressure and the duration of having diabetes $(\mathrm{r}=0.46$, $\mathrm{P}=0.03$ ). In other words, the longer the duration of disease and higher the level of $\mathrm{HbA} 1 \mathrm{c}$, the more significant the reduction of end-tidal carbon dioxide pressure is. The results of the current study were in line with the findings of the study by Zindeldin et al. [19]. In their study conducted on 45 patients with diabetes, a strong and inverse relationship was observed between diminished pulmonary volumes and the duration of diabetes $(r=-0.88)$.

In other words, improper control of blood sugar is associated with diminished volume of the lung, which is more common in the patients with a longer duration of diabetes. In a similar and prospective study with oneyear follow-up, Ford et al. indicated that the low forced vital capacity and the forced expiratory volume of air in the first second are associated with the incidence of diabetes. In other words, low end-tidal carbon dioxide pressure itself is a factor for elevated blood sugar levels [23].

Animal studies suggested that hypoxia reduced insulin sensitivity and predisposed the animals to type 2 diabetes [24]. The current study had some limitations. The lung functions of patients were investigated in the

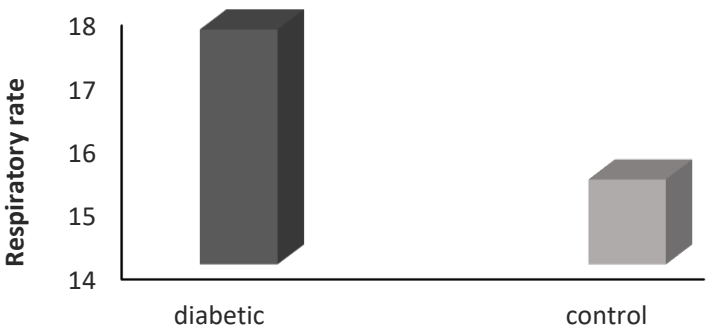

Figure 1. Comparison of end tidal carbon dioxide pressure and respiratory rate between two groups 
nonfunctional sitting position. The second challenge was the extreme sensitivity of the device to the humidity of the environment and the need to replace the adapter frequently. Accordingly, it is suggested that in future studies, the respiratory indicators of diabetic patients be investigated during a dynamic activity.

The results of the current study indicated diminished end-tidal carbon dioxide pressure and increased respiration rate in patients with type 2 diabetes, compared to healthy individuals. Also, an inverse and strong significant relationship was observed between $\mathrm{HbA} 1 \mathrm{c}$ and endtidal carbon dioxide pressure, while an inverse and moderate correlation was found between end-tidal carbon dioxide pressure and the duration of having diabetes. Based on the results of the current study, a respiration therapeutic program should be considered to facilitate and prevent impaired pulmonary function for such patients.

\section{Ethical Considerations}

Compliance with ethical guidelines

The study proposal was approved by the Research Council of Rehabilitation Sciences Faculty of Ahvaz Jundishapour University of Medical Sciences (ethical code: IR.AJUMS. REC.1396.375).

Funding

This paper was extracted from the MSc. thesis of first author, in Research Center for Musculoskeletal Rehabilitation, Ahvaz Jundishapur University of Medical Sciences (No. PHT-9609).

Authors' contributions

All authors contributed in preparing this article.

\section{Conflict of interest}

The authors declared no conflict of interest.

Acknowledgments

The authors highly appreciate the support of the Vice Chancellor for Research and Technology at the University for their financial aids.

\section{References}

[1] Anandhalakshmi S, Manikandan S, Ganeshkumar P, Ramachandran C. Alveolar gas exchange and pulmonary functions in patients with type II diabetes mellitus. Journal of Clinical and Diagnostic Research. 2013; 7(9):1874-77. [DOI:10.7860/JCDR/2013/6550.3339] [PMID] [PMCID]

[2] Forbes JM, Cooper ME. Mechanisms of diabetic complications. Physiological Reviews. 2013; 93(1):137-88. [DOI:10.1152/physrev.00045.2011] [PMID]

[3] Hamdy G, Amin M, Rashad A. Pulmonary function changes in diabetic lung. Egyptian Journal of Chest Diseases and Tuberculosis. 2013; 62(3):513-7. [DOI:10.1016/j.ejcdt.2013.07.006]

[4] Vojtková J, Čiljaková M, Michnová Z, Turčan T. Chronic complications of diabetes mellitus related to the respiratory system. Pediatric Endocrinology, Diabetes \& Metabolism. 2012; 18(3):112-5. [PMID]

[5] Kerner W, Brückel J. Definition, classification and diagnosis of diabetes mellitus. Experimental and Clinical Endocrinology \& Diabetes. 2014; 122(7):384-6. [DOI:10.1055/s-0034-1366278] [PMID]

[6] Courtney R. The functions of breathing and its dysfunctions and their relationship to breathing therapy. International Journal of Osteopathic Medicine. 2009; 12(3):78-85. [DOI:10.1016/j.ijosm.2009.04.002]

[7] Perri MA, Halford E. Pain and faulty breathing: A pilot study. Journal of Bodywork and Movement Therapies. 2004 8(4):297-306. [DOI:10.1016/S1360-8592(03)00085-8]

[8] McLaughlin L. Breathing evaluation and retraining in manual therapy. Journal of Bodywork and Movement Therapies. 2009; 13(3):276-82. [DOI:10.1016/j.jbmt.2009.01.005] [PMID]

[9] Pitocco D, Fuso L, Conte EG, Zaccardi F, Condoluci C, Scavone G, et al. The diabetic lung-a new target organ. The Review of Diabetic Studies. 2012; 9(1):23-35. [DOI:10.1900/ RDS.2012.9.23] [PMID] [PMCID]

[10] Shah SH, Sonawane P, Nahar P, Vaidya S, Salvi S. Pulmonary function tests in type 2 diabetes mellitus and their association with glycemic control and duration of the disease. Lung India. 2013; 30(2):108-12. [DOI:10.4103/0970-2113.110417] [PMID] [PMCID]

[11] Klein OL, Krishnan JA, Glick S, Smith LJ. Systematic review of the association between lung function and Type 2 diabetes mellitus. Diabetic Medicine. 2010; 27(9):977-87. [PMID]

[12] Dharwadkar AR, Dharwadkar AA, Banu G, Bagali SH. Reduction in lung functions in type-2 diabetes in Indian population: Correlation with glycemic status. Indian Journal of Physiology and Pharmacology. 2011; 55(2):170-5. [PMID]

[13] Singh J, Gupta KK, Himanshu D, Dinkar A, Atam V, Kant $S$. To study the effect of glycemic control and duration of disease on pulmonary function tests and diffusion capacity in type 2 diabetes mellitus. International Journal of Research in Medical Sciences. 2015; 3(1):224-8. [DOI:10.5455/2320-6012. ijrms20150140]

[14] Schallom L, Ahrens T. Hemodynamic applications of capnography. Journal of Cardiovascular Nursing. 2001; 15(2):5670. [PMID] 
[15] Pellegrino R, Viegi G, Brusasco V, Crapo RO, Burgos F, Casaburi RE, et al. Interpretative strategies for lung function tests. European Respiratory Journal. 2005; 26(5):948-68. [DOI: 10.1183/09031936.05.00035205] [PMID]

[16] Chhajed PN, Miedinger D, Baty F, Bernasconi M, Heuss LT, Leuppi JD, et al. Comparison of combined oximetry and cutaneous capnography using a digital sensor with arterial blood gas analysis. Scandinavian Journal of Clinical and Laboratory Investigation. 2010; 70(1):60-4. [DOI:10.3109/00365510903450106] [PMID]

[17] Monnier L, Lapinski H, Colette C. Contributions of fasting and postprandial plasma glucose increments to the overall diurnal hyperglycemia of type 2 diabetic patients: variations with increasing levels of HbA1c. Diabetes Care. 2003; 26(3):881-5. [PMID]

[18] Ravanbakhsh M, Nargesi M, Raji H, Shoushtari MH. Reliability and validity of the Iranian version of Nijmegen questionnaire in Iranians with asthma. Tanaffos. 2015; 14(2):121-7. [PMID] [PMCID]

[19] Zineldin MA, Hasan KA, Al-Adl AS. Respiratory function in type II diabetes mellitus. Egyptian Journal of Chest Diseases and Tuberculosis. 2015; 64(1):219-23. [DOI:10.1016/j. ejcdt.2014.08.008]

[20] Fuso L, Pitocco D, Condoluci C, Conte E, Contu C, Rizzi A, et al. Decline of the lung function and quality of glycemic control in type 2 diabetes mellitus. European Journal of Internal Medicine. 2015; 26(4):273-8. [PMID]

[21] Wheatley CM, Baldi JC, Cassuto NA, Foxx-Lupo WT, Snyder EM. Glycemic control influences lung membrane diffusion and oxygen saturation in exercise-trained subjects with type 1 diabetes. European Journal of Applied Physiology. 2011; 111(3):567-78. [DOI:10.1007/s00421-010-1663-8] [PMID]

[22] Pu LJ, Shen Y, Lu L, Zhang RY, Zhang Q, Shen WF. Increased blood glycohemoglobin A1c levels lead to overestimation of arterial oxygen saturation by pulse oximetry in patients with type 2 diabetes. Cardiovascular Diabetology. 2012; 11(1):110. [PMID] [PMCID]

[23] Ford ES, Mannino DM. Prospective association between lung function and the incidence of diabetes: Findings from the National Health and Nutrition Examination Survey epidemiologic follow-up study. Diabetes Care. 2004; 27(12):296670. [PMID]

[24] Yeh HC, Punjabi NM, Wang NY, Pankow JS, Duncan BB, Brancati FL. Vital capacity as a predictor of incident type 2 diabetes: The atherosclerosis risk in communities study. Diabetes Care. 2005; 28(6):1472-9. [PMID] 